pour l'étude des processus fermentatifs et pour l'utilisation des ferments sélectionnés soit dans la laiterie soit dans l'ensilage des fourrages.

La production microbienne enzymatique est génotypique, réglée par les lois de l'hérédité. Partant, chaque espèce, tout en possédant une constante d'enzymes déterminées, présente aussi une variabilité enzymatique, par divergences individuelles déterminées, toujours dans le cadre du génotype, sans modification de l'idioplasme.

\title{
BIBLIOGRAPHIE
}

[1] Rend. Istit. Lomb. Sc. Lett., 1939, LXXIII et 1943-1944, LXXVII.

[2] Rend. Istit. Lomb. Sc. Lett., 1921, LIV ; Le Lait, 1922, II, 2.

[3] Rend. Istit. Lomb. Sc. Lett., 1920, LVII et 1940-1941, LXXIV.

[4] Comptes Rendus Acad. Sc., 22 décembre 1924 et 16 novembre 1925 : Le Lait, 1926, VI, 81.

[5] Landw. Jahrb. der Schweiz, 1941, 176.

[6] Annales Microb., 1942, II.

[7] Journal of Dairy Sc., 1937, XX.

[8] Le Lait, 1937, XVII, 963 et 1938, XVIII, 174.

[9] Journal Bact., 1938, XXXVI.

[10] Rend. Istit. Lomb. Sc. Lett., 1931, LXIV ; Le Lait, 1930, X, 745.

[11] Rend. Istit. Lomb. Sc. Lett., 1923, LVI et 1927, LX ; Le Lait, 1924, IV, 382 et 1928 , VIII, 465.

[12] Landw. Jahrb. der Schweiz, 1941, 407.

\section{DOSAGE DE LA MATIÈRE GRASSE DES CRËMES NOTES POUR UNE ETUdE RATIONNELLE DES MÉTHOdES ACIDO-BUTYROMÉTRIQUES}

\author{
par \\ PAUL CADOR \\ Ingénieur Agronome \\ Industriel Laitier
et PIERre MACHEREL
Ingénieur Agricole \\ Diplômé de la S. E. S. I. L. \\ Ingénieur technique aux Etablissements \\ laitiers Mallet, Alençon
}

\section{INTRODUCTION - PLAN DE TRAVAIL}

L'analyse des crèmes par les procédés acido-butyrométriques peut schématiquement se diviser en deux séries d'opérations :

10 Une séparation de la matière grasse sous l'influence de facteurs chimiques et physiques ;

$2^{\circ}$ Des opérations de mesures et de calculs :

Mesure de la quantité de crème introduite, 
Mesure du volume occupé par la matière grasse séparée;

Calcul pour déduire de ces deux mesures la teneur de l'échantillon analysé en poids de matière grasse contenue dans un certain poids de crème.

Pour bien comprendre les méthodes et les bien /utiliser, il est nécessaire de les décomposer et de les étudier en chacun de leurs éléments.

Dans une première partie, nous avons tenté d'exposer une théorie de ces méthodes et d'établir des axes de recherches. Une deuxième partie est consacrée aux mesures de laboratoire. Elle prétend plus proposer des méthodes d'investigation que poser des résultats définitifs, que seul un long travail en commun pourrait établir solidement.

\section{PREMIÈRE PARTIE \\ ETUDE THEORIQUE DES MÉTHODES \\ I. SÉPARATION DE LA MATIERE QRASSE}

\section{A. Notion de densité apparente de la colonne grasse}

D'une certaine quantité $P$ de crème nous séparons, par un processus physico-chimique défini, un produit gras représentant la matière grasse contenue dans $P$.

La séparation de la matière grasse telle qu'elle résulte des données d'une méthode peut être complète ou incomplète ; la matière grasse ainsi séparée peut être pure ou contenir des impuretés.

On peut la caractériser par un rapport :

Quantité de matière grasse pure contenue dans la crème

$$
\text { Quantité de corps gras extrait }
$$

Par exemple, le rapport :

Poids de matière grasse pure contenue dans l'échantillon

Poids du corps gras extrait

est égal à 1 si toute la matière grasse est extraite et ne contient pas d'impuretés, ou le rapport :

Poids de matière grasse pure contenue dans l'échantillon

$\overline{\text { Volume du corps gras extrait dans les conditions de lecture }}$

est égal à la densité de la matière grasse pure dans les conditions de lecture si toute la matière grasse est extraite et ne contient pas d'impuretés et dans tous les cas égal à un certain chiffre "dà que nous appelons densité apparente par analogie. C'est ce rapport que 
nous avons adopté pour caractériser la séparation de la matière grasse dans la technique employée.

La densité apparente de la matière grasse extraite d'une crème par la technique d'analyse employée est égale pour chaque extraction au rapport du nombre exprimant le poids de matière grasse pure contenu dans l'échantillon au nombre exprimant le volume du corps gras séparé de ce même échantillon dans l'opération considérée (volume et densité étant pris dans les conditions de lecture).

da caractérise la séparation de la matière grasse de la crème (et non pas seulement la matière grasse séparée) sa valeur dépendant évidemment du processus physico-chimique de l'extraction.

\section{B. Eléments de la densité apparente. Valeurs particulières de da}

\section{a) Eléments de la densité apparente.}

Si p représente le poids de matière grasse pure contenu dans l'échantillon,

p' le poids de corps gras extrait dans l'opération,

d' la densité du corps gras dans les conditions de lecture :

$$
d a=\frac{p}{p^{\prime}} d^{\prime}
$$

Si $p_{1}$ représente le poids de matière grasse pure contenu dans 1 gramme de corps gras séparé,

$\mathrm{p}_{2}$ le poids de matière grasse pure extrait pour 1 gramme de matière grasse pure contenu dans l'échantillon :

$$
\begin{aligned}
\mathrm{p} \times \mathrm{p}_{2} & =\mathrm{p}^{\prime} \times \mathrm{p}_{1} \\
\frac{\mathrm{p}}{\mathrm{p}^{\prime}} & =\frac{\mathrm{p}_{1}}{\mathrm{p}_{2}} \\
\mathrm{da} & =\frac{\mathrm{p}_{1}}{\mathrm{p}_{2}} \mathrm{~d}^{\prime} \quad \text { équation } \mathrm{n}^{\circ} 1
\end{aligned}
$$

\section{b) Valeurs particulières de da.}

Si toute la matière grasse est extraite et ne contient pas d'impuretés et si d est la densité de la matière grasse pure :

$$
\left.\begin{array}{l}
\mathrm{p}_{1}=1 \\
\mathrm{p}_{2}=1 \\
\mathrm{~d}^{\prime}=\mathrm{d}
\end{array}\right\} \mathrm{da}=\mathrm{d}
$$

Si toute la matière grasse est extraite et contient des impuretés :

$$
\mathrm{p}_{2}=1 \quad \mathrm{da}=\mathrm{d}^{\prime} \mathrm{p}_{1}
$$


Si une partie seulement de la matière grasse est extraite et si cette partie est pure :

$$
\left.\begin{array}{l}
\mathrm{P}_{1}=1 \\
\mathrm{~d}^{\prime}=\mathrm{d}
\end{array}\right\} \mathrm{da}=\frac{\mathrm{d}}{\mathrm{p}_{2}}
$$

\section{Importance de da. Valeur et constance}

da caractérise la première série d'opérations "séparation de la matière grasse sous l'influence de facteurs physiques et chimiques » et représente le résultat du processus physico-chimique d'extraction dans les calculs donnant le résultat. Il relie les deux séries d'opérations et se présente comme la clef de voûte de l'ensemble (voir schéma p. 208).

Pour que la méthode soit d'application possible il est évident que da doit avoir une valeur pratiquement constante dans des conditions définies et contrôlables.

Il est permis de douter à priori de la constance de da.

D'après M. Pien (Le Lait, no 176) la densité de la matière grasse pure extraite des produits fermentés est supérieure à celle de la matière grasse pure extraite des produits frais ce qui (toutes choses égales par ailleurs) augmenterait la densité apparente dans les analyses de crèmes acides (1).

La détermination de la valeur de da, des lois présidant à ses variations éventuelles ne peut être faite qu'expérimentalement. Des méthodes d'investigation ont été discutées dans la deuxième partie de cette étude, mais da étant posé la seconde série d'opéra. tions ne comporte plus que des mesures et des calculs théoriquement simples et doit donc être susceptible d'une précision mathématique.

\section{MESURES ET CALCULS}

\section{A. Etablissement de l'équation générale donnant le teneur}

La définition que nous avons donnée de da nous permet de poser l'équation générale donnant la teneur.

Si V représente le volume de corps gras extrait,

Tla teneur en poids \%,

(1) Les phénomènes qui président à la séparation sont très mal connus aussi est-il imprudent d'admettre sans vérification que da est constant et indépendant de la teneur pour des crèmes prises au même degré d'acidité en particulier pour les crèmes fraîches.

Etablissant nos calculs en partánt de cette hypothèse à priori contestable, l'étude des graduations des butyromètres nous montrera qu'elle a été, sinon consciemment du moins en fait, admise par les principaux constructeurs d'appareils en service. Sous réserve de vérifications expérimentales, nous admettons aussi pour l'étude théorique que la densité apparente $a$, pour les crèmes fraîches, une valeur constanté que nous désignerons par dao 
P le poids de crème introduit dans l'appareil,

da la densité apparente de la séparation considérée :

$$
\mathbf{T}=\frac{V d \mathbf{a} \times 100}{\mathrm{P}}
$$

Mais le volume $V$ est évalué par la lecture des graduations d'une tige de verre où se rassemble le corps gras extrait.

Par définition la lecture est déterminée par le plan horizontal tangent au ménisque de matière grasse. Toute la matière grasse qui se trouve all-dessus de ce plan, dans ce que nous appelons "les cornes du ménisque" n'est pas comprise dans cette lecture.

Soit $\mathrm{m}$ le volume des cornes,

$\checkmark$ le volume compris entre le 0 et la graduation correspondant au plan tangent au ménisque gras.

Nous arons $V=v+m$, et l'équation générale donnant la teneur devient :

$$
T=\frac{(v+m) d a \times 100}{P}
$$

m peut être considéré comme pratiquement constant pour un type de butyromètre déterminé.

da est supposé déterminé. (Dans le cas des crèmes fraîches par hypothése da $=$ dao.) T.

Une mesure de $\mathbf{v}$ et de $\mathrm{P}$ et un simple calcul permettent d'obtenir

\section{B. Cas particuliers. Obtention de la teneur par lecture directe sans calcul}

Dans le cas des crèmes fraîches nous avons supposé da $=$ dao $=$ constante.

Si dans l'équation $\mathrm{n}^{\circ} 2$ nous donnons à $\mathrm{P}$ une valeur fonction de $T$ ou une valeur constante, $v$ sera aussi fonction de $T$ et le butyro mètre pourra être gradué directement en teneurs.

\section{a) Méthode volumétrique originale Koehler.}

1. Définition du butyromètre Koehler original.

Les butyromètres pour l'analyse de la crème selon la méthode du $\mathrm{D}^{\mathrm{r}}$ Koehler ont été conçus pour recevoir $5 \mathrm{~cm}^{3}$ de crème fraîche et donner les résultats par simple lecture en grammes de matière grasse pure pour 100 grammes de crème.

2. Etude de la graduation. Variation de la fonction $\mathrm{v}=\mathrm{f}(\mathrm{T})$. 
Par définition $\mathrm{P}$ est le poids de $5 \mathrm{~cm}^{3}$ de crème, si $\mathrm{D}$ est la densité de cette même crème nous avons :

$$
\mathrm{P}=5 \mathrm{D}
$$

Or la densité de la crème fraîche diminue quand sa teneur en matière grasse augmente. HunzIKen dans "The Butter Industry " (page 561) a établi un tableau de ces variations. Elles se traduisent graphiquement entre 10 et 60 par une courbe très tendue (voir graphique) qu'on peut assimiler à une droite dont l'équation est :

$$
\mathrm{D}=0,00108 \mathrm{~T}+1,0343
$$

L'équation no 2 devient :

$$
\mathrm{T}=\frac{(\mathrm{v}+\mathrm{m}) \mathrm{dao} \times 100}{-0,0054 \mathrm{~T}+5,1715}
$$

d'où :

$$
\mathrm{v}+\mathrm{m}=\frac{-0,0054 \mathrm{~T}^{2}+5,1715 \mathrm{~T}}{100 \text { dao }} \quad \text { équation } \mathrm{n}^{0} 3
$$

L'équation no 3 est de la forme :

$$
v=-a T^{2}+b T-m \quad \text { équation } n^{\circ} 3 \text { bis }
$$

$a$ et $b$ étant positifs et constants.

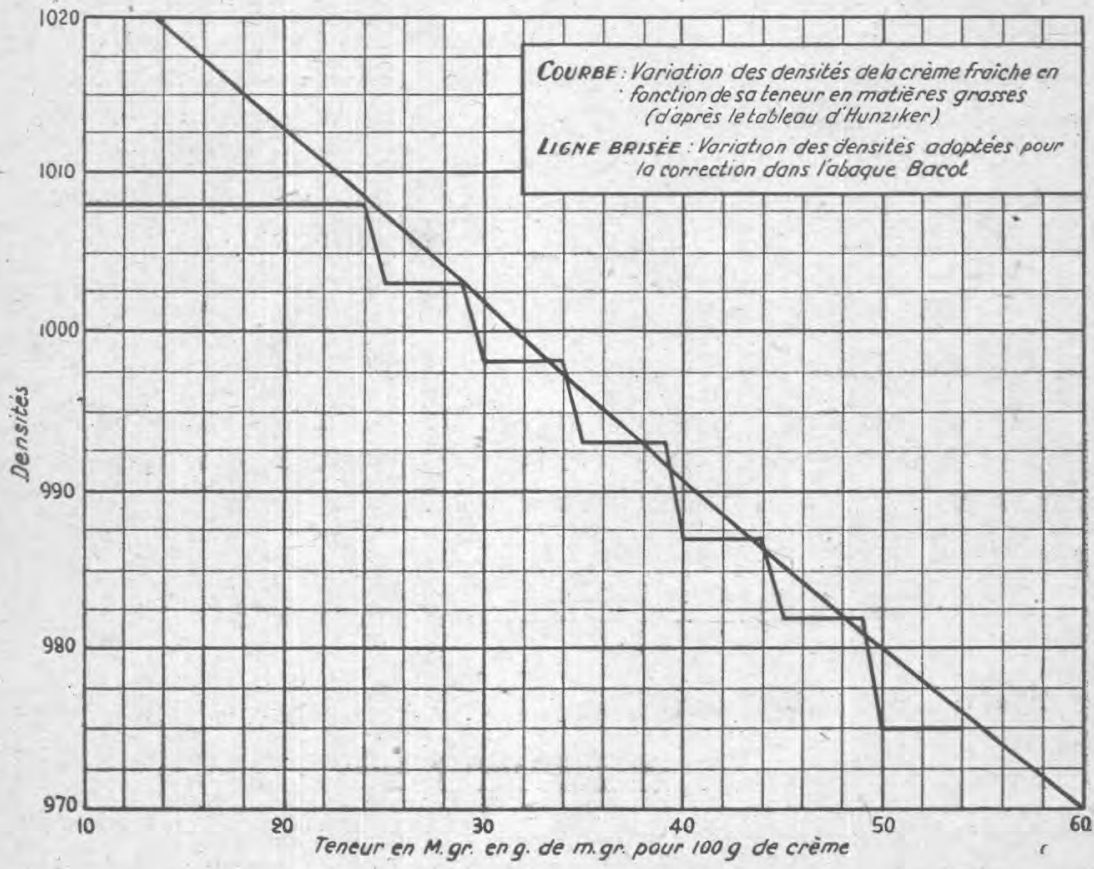

Graphique 1 
$\mathbf{v}$ est une fonction de $\mathbf{T}$ et le butyromètre peut être directement gradué en teneurs, les résultats seront obtenus par simple lecture.

Si $\nabla_{1}$ est le volume compris entre le 0 et une graduation quel conque $\mathrm{T}_{1}$, et $v_{2}$ le volume compris entre le 0 et une autre graduation $T_{2}\left(T_{2}>T_{1}\right.$. Le volume compris entre ces deux graduations $\mathrm{T}_{2}$ et $\mathrm{T}_{1}$ est égal à :

$$
\begin{gathered}
v_{2}-v_{1}=-a\left(T_{2}-T_{1}\right)+b\left(T_{2}-T_{1}\right) \\
v_{2}-v_{1}=-a\left(T_{2}+T_{1}\right)\left(T_{2}-T_{1}\right)+b\left(T_{2}-T_{1}\right) \\
\frac{v_{2} v_{1}}{T_{2}-T_{1}}=-a\left(T_{2}+T_{1}\right)+b \\
\frac{v_{2}-v_{1}}{T_{2}-T_{1}}=-2 a \frac{T_{2}+T_{1}}{2}+b
\end{gathered}
$$

Si $T_{2}-T_{1}=1$ (cas de deux graduations consécutives),

$v_{2}-v_{1}$ est le volume $v^{\prime}$ d'une graduation correspondant à une teneur 'T' telle que :

$$
\mathrm{T}^{\prime}=\frac{\mathrm{T}_{2}+\mathrm{T}_{1}}{2}
$$

et nous avons $v^{\prime}=-2 a T^{\prime}+b$.

En conclusion : Si da est indépendant de la teneur et constant pour les crèmes fraîches; dans un butyromètre Koehler gradué rigoureusement le volume délimité par 2 graduations consécutives sera d'autant plus petit que ces graduations seront voisines de la graduation 60 (1).

La section de la tige du butyromètre étant constante sur toute sa longueur par construction, cette variation de vo lume se traduira par une variation de l'intervalle entre les graduations.

3. Emploi de la méthode Koehler originale.

\section{a) Crèmes fraîches.}

Ayant introduit dans le butyromètre $5 \mathrm{~cm}^{3}$ d'une crème de teneur $\mathrm{T}$ et de densité $\mathrm{D}$ le volume occupé par le corps gras sera égal à $V_{1}=v_{1}+m$ et la teneur lue à $T_{1}$ :

avec :

$$
\mathrm{T}_{1}=\frac{\left(\mathrm{v}_{1}+\mathrm{m}\right) \text { dao } \times 100}{5 \mathrm{D}_{1}} \text { par construction }
$$

La teneur réelle $\mathrm{T}$ sera égalè à :

$$
\mathbf{T}=\frac{\left(v_{1}+\mathrm{m}\right) \mathrm{dao} \times 100}{5 \mathrm{D}}
$$

(1) Le volume compris entre 0 et 10 peut être plus faible que celui entre 10 et 20 car la correction due au volume $m$ du ménisque porte sur la place de la graduation 0. 
Et nous avons :

$$
\mathrm{DT}=\mathrm{D}_{1} \mathrm{~T}_{1}
$$

en remplaçant dans les deux membres dé l'équation, D par sa valeur en fonction de $\mathrm{T}$; d'après la courbe d'Hunziker il vient :

$$
\begin{gathered}
(0,00108 \mathrm{~T}+1,0343) \mathrm{T}=\left(0,00108 \mathrm{~T}_{1}+1,0343\right) \mathrm{T}_{1} \\
0,00108\left(\mathrm{~T}^{2}-\mathrm{T}_{1}^{2}\right)+1,0343\left(\mathrm{~T}_{1}-\mathrm{T}_{1}\right)=0 \\
{[0,00108(\mathrm{~T}+\mathrm{T})+1,0343](\mathrm{T}-\mathrm{T})=0}
\end{gathered}
$$

Equation qui n'est vérifiée que pour $\mathrm{T}_{1}=\mathrm{T}$.

La teneur lue correspond à la teneur réelle.

\section{b) Crèmes fermentées.}

$$
\begin{aligned}
& \text { Teneur lue }=T_{1}=\frac{\left(v_{1}+m\right) \text { dao } \times 100}{5 D_{1}} \\
& \text { Teneur réelle } T=\frac{\left(v_{1}+m\right) d a \times 100}{5 D^{\prime}}
\end{aligned}
$$

Dans une crème fermentée, plus ou moins gazeuse, il n'y a pas de relation définie entre la teneur en matière grasse et la densité; pas de relation entre $D^{\prime}$ et $T$.

Si D représente la densité correspondant à une crème fraîche de teneur T et $D^{\prime}$ la densité de la même crème fermentée ; nous arons $\mathrm{D}^{\prime}<\mathrm{D}$

$$
\begin{gathered}
\mathrm{D}^{\prime}=\mathrm{D}-\varepsilon \\
\varepsilon>0 \\
\frac{\mathrm{T}}{\mathrm{T}_{1}}=\frac{\mathrm{da} \mathrm{D}_{1}}{\mathrm{dao} \mathrm{D}^{\prime}} \\
\frac{\mathrm{T}}{\mathrm{T}_{1}}=\frac{\mathrm{da} \mathrm{D}_{1}}{\mathrm{dao}(\mathrm{D}-\varepsilon)} \\
\frac{\mathrm{T}(\mathrm{D}-\varepsilon)}{\mathrm{T}_{1} \mathrm{D}_{1}}=\frac{\mathrm{da}}{\mathrm{dao}}>1 \\
\frac{\mathrm{TD}-\mathrm{T} \varepsilon}{\mathrm{T}_{1} \mathrm{D}_{1}}=\frac{\mathrm{da}}{\mathrm{dao}}>1 \\
\frac{0,00108 \mathrm{~T}^{2}+(1,0343-\varepsilon) \mathrm{T}}{0,00108 \cdot \mathrm{T}_{1}^{2}+1,0343 \mathrm{~T}_{1}} 1
\end{gathered}
$$

relation qui ne peut évidemment être vérifiée qu'avec $\mathbf{T}>\mathbf{T}_{\mathbf{1}}$. La méthode volumétrique originale employée aveo des crèmes fermentées tend donc à donner des résultats trop faibles. 


\section{b) Méthode pondérale volumétrique Roeder (1)}

\section{Définition du butyromètre Roeder.}

Les butyromètres pour l'analyse de la erème selon la méthode du $\mathrm{D}^{\mathrm{r}}$ Roeder ont été conçus pour recevoir 5 grammes de crème et donner les résultats par simple lecture en grammes de matière grasse pour 100 grammes de crème.

2. Etude de la graduation.

Par définition $\mathrm{P}=\mathbf{5}$.

L'équation $n^{\circ} 2$ devient :

$$
\begin{aligned}
T & =\frac{(\mathrm{v}+\mathrm{m}) \text { dao } \times 100}{5} \\
& \mathrm{v}=\mathrm{T} \frac{5}{100 \text { dao }}-\mathrm{m} \quad \text { équation } \mathrm{n}^{\circ} 4
\end{aligned}
$$

$\checkmark$ est une fonction linéaire de T. Le butyromètre peut être gradué en teneurs ; les résultats peuvent être obtenus par lecture directe.

Si $v_{1}$ représente le volume compris entre le 0 et une graduation quelconque $\mathrm{T}_{1}$.

$\nabla_{2}$ le volume compris entre le 0 et une autre graduation $\mathrm{T}_{2},\left(\mathrm{~T}_{2}>\mathrm{T}_{1}\right)$.

Le volume compris entre les graduations $T_{1}$ et $T_{2}$ sera égal à :

$$
\nabla_{2}-\nabla_{1}=\frac{5}{100 \text { dao }}\left(T_{2}-T_{1}\right)
$$

et si $T_{2}-T_{1}=1$, cas de deux graduations consécutives.

$v_{2}-v_{1}=v^{\prime}$ volume compris entre deux graduations consécutives. avec $v^{\prime}=\frac{1}{20 \text { dao }}$ qui est constant.

Par conséquent :

Si «da» est constant, dans un butyromètre Roeder (ou dans tout autre butyromètre où il faut introduire un poids déterminé de crème) gradué rigoureusement, 2 graduations consécutives limitent un volume constant (2).

(1) La méthode Roeder pondérale volumétrique peut être dans son principe général (extraction-mesures) rapprochée des méthodes Koehler et dérivées. II suffira de savoir que dao et $\mathrm{m}$ ont pour cette méthode une valeur partieulière différente.

(2) Les butyromètres Roeder gradués à l'équidistance ont néanmoins entre 0 et. 7 un intervalle non gradué qui tient compte de la correction due au volume $\mathrm{m}$ des cornes du ménisque

Si vo désigne le volume compris entre 0 et 10

v 0 le volume constant de 10 graduations entre 10 et 70

$\mathrm{m}=\mathrm{v} 10 \cdot \mathrm{v} 0$. 


\section{Emploi de la méthode Roeder.}

\section{a) Crèmes fralches.}

Chaque graduation entre 7 et 70 a un volume égal à $\frac{1}{20 \text { dao }}$ Le volume compris entre 0 et 7 est égal à $\frac{7}{20 \text { dao }}-\mathrm{m}$.

Une lecture de $T_{1}$ correspond à un volume gras de :

soit :

$$
\frac{7}{20 \text { dao }}-m+\left(T_{1}-7\right) \frac{1}{20 \text { dao }}+m
$$

$$
\frac{T_{1}}{20 \text { dao }}
$$

c'est-à-dire à un poids de matière grasse pure de :

$\frac{T_{1}}{20}$ dans 5 grammes d'échantillon et de $T_{1}$ dans 100 grammes

La teneur réelle $\mathbf{T}=\mathbf{T}_{1}$ la teneur lue.

\section{b) Crèmes acides.}

La teneur lue $\mathrm{T}_{1}$ correspond à un volume de :

$$
\frac{\mathrm{T}_{1}}{20 \text { dao }} \text { de corps gras extrait, }
$$

soit à un poids de matière grasse pure de :

$$
\begin{aligned}
& \frac{T_{1}}{20} \times \frac{\text { da }}{\text { dao }} \text { dans } 5 \text { grammes d'échantillon et de: } \\
& T_{1} \times \frac{d a}{\text { dao }} \text { dans } 100 \text { grammes. } \\
& T=T_{1} \times \frac{d a}{d a o}
\end{aligned}
$$

La teneur réelle $T=T_{1}$ la teneur lue si $\frac{d a}{d a o}=1$.

(suite de la note (2) de la page 204).

Nous avons par ailleurs v $10=\frac{1}{2 \text { dao }}$

L'étalonnage des butyromètres Roeder permet donc de déterminer facilement les chiffres adoptés par le constructeur pour le volume $\mathrm{m}$ des cornes du mnisque et la densité apparente dao. 


\section{Cas général. Mesures et calcul}

Si au lieu d'une quantité déterminée d'échantillon nous introduisons un poids quelconque $\mathrm{P}$ de crème dans le butyromètre un simple calcul (ou la lecture d'une abaque à double entrée) nous donnera $T$. La tige du butyromètre ne sert que comme appareil de mesure de capacité et peut être assimilée à une pipette graduée. Les volumes délimités par les graduations peuvent être quelconques, il suffit que ces volumes soient exactement connus. On pourra done utiliser aussi bien les butyromètres Koehler originaux que tels butyromètres à graduations équidistantes.

a) Utilisation des butyromètres Koehler originaux pour résoudre le cas général, méthode pondérale volumétrique Koehler-Bacot.

Introduisons dans un butyromètre Koehler original un poids quelconque de crème $\mathbf{P}$.

La teneur réelle $T$ est donnée par l'équation :

$$
\mathrm{T}=\frac{\left(\nabla_{1}+\mathrm{m}\right) \mathrm{da} \times 100}{\mathrm{P}}
$$

$\nabla_{1}$ désignant le volume de matière grasse compris entre les graduations,

da la densité apparente de l'extraction considérée.

La teneur lue $\mathrm{T}_{1}$ est la teneur correspondant à $\nabla_{1}$ qui par construction est égale à :

$$
\mathrm{T}_{1}=\frac{\left(\mathrm{v}_{1}+\mathrm{m}\right) \mathrm{dao} \times 100}{5 \mathrm{D}_{1}}
$$

Des équations précédentes, nous tirons :

$$
\mathrm{T}=\mathrm{T}_{1}^{\frac{5 \mathrm{D}^{1}}{\mathrm{P}} \times \frac{\mathrm{da}}{\text { dao }}} \quad \text { équation } \mathrm{n}^{0} 5
$$

1. Cas de crèmes frâ̂ches.

$$
\begin{aligned}
& \mathrm{da}=\mathrm{dao} \\
& \mathrm{T}=\mathrm{T}_{1}-\frac{5 \mathrm{D}_{1}}{\mathrm{P}}
\end{aligned}
$$

Formule qui a servi de principe à la construction de l'abaque de M. Bacot, elle est exacte pour les crèmes fraîches si les butyromètres Koehler originaux sont exactement gradués, si la densité apparente est une constante pour les crèmes fraîches et si le $D_{1}$ 
adopté pour la correction correspond bien au $\mathrm{D}_{1}$ de la graduation (et non de l'essai) (1).

2. Cas de crèmes fermentées.

Si da $>$ dao, toutes choses justes par ailleurs la formule Bacot donnerait des résultats faibles.

\section{b) Utilisation de butyromètres gradués à l'équidistance pour résoudre le cas général.}

Soit $\nabla^{\prime}$ le volume délimité entre 2 graduations consécutives. La teneur lue $T_{1}$ correspond à un volume :

$$
\mathrm{V}=\mathrm{T}_{\mathbf{1}} \mathrm{v}^{\prime}+\mathrm{m}
$$

et donc à un poids de matière grasse pure dans l'échantillon égal à $\left(\mathrm{T}_{1} \mathrm{v}^{\prime}+\mathrm{m}\right)$ da.

La teneur réelle est donnée par la formule :

$$
\mathrm{T}=\mathrm{T} \frac{\left.\mathrm{T} \mathrm{v}^{\prime}+\mathrm{m}\right) \mathrm{da} \times 100}{\mathrm{P}} \quad \text { équation } \mathrm{n}^{\circ} 6
$$

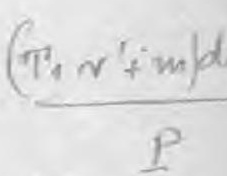

Pour établir une abaque de correction rigoureuse il s'agit seulement de déterminer $\mathrm{m}$, da et la loi de ses variations.

\section{c) Utilisation de butyromètres à graduations particulières.}

Il est possible aussi d'établir une abaquẻ de correction rigoureuse pour l'emploi de tel type déterminé de butyromètre " Koehler " même non rigoureusement gradués, à condition de déterminer $\mathrm{m}$ et da et de mesurer les volumes correspondant aux diverses teneurs lues. La formule $\mathrm{n}^{0} 2$ donnera toujours le résultat exact :

$$
\mathrm{T}=\frac{(\mathrm{v}+\mathrm{m}) \mathrm{da} \times 100}{\mathrm{P}}
$$

\section{CONCLUSION DE L'ÉTUdE THEORIQUE}

La mise en évidence de la densité apparente, clef de voûte, de l'ensemble et la distinction des deux séries d'opérations est abso lument nécessaire à une étude sérieuse de la méthode. Jusqu'à présent les chimistes ne séparant pas nettement ces éléments se sont contentés de comparer les résultats derniers de la méthode avec ceux d'une méthode de référence, ce qui ne permet pas d'isoler les causes d'erreur. La comparaison doit s'effectuer au niveau de da

(1) En pratique si l'on porte sur un graphique la variation des densités adoptées par M. BACor pour la construction de son tableau de correction, on a une ligne brisée en escalier, différente, par sa forme même de la courbe très tendue qui représente les variations de la densité de la crème fraîche en fonction des teneurs en matière grasse (voir graphique p. 201). 
Schema résumant l'étude théorique

I-Mesure de la
quantité
d'échantilion

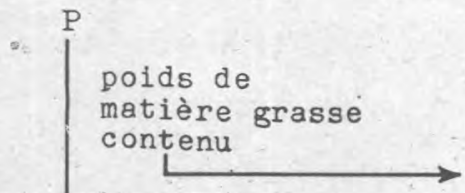

II-Evaluation

de la teneur

-Equation générale
III-Mesure du

volume gras séparé volume gras

séparé dans un

tube gradué

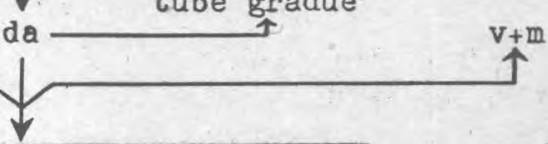

$T=\underbrace{(v+m) d a \times I 00}_{P}$
Résultat obtenu sans calcul

$P$ déterminé

Méthode Koehler Méthode

volumétrique Roeder

originale

$P=5 D=P \quad(T)$

crème crème

fraiche acide

$\mathrm{d} a=\mathrm{dao} \quad \mathrm{d} a=\mathrm{dao}$

$P=f(T) P=f(T)$

$\mathbf{v}=f(T)$

pondérale
Cas général:Mesures

et calculs

résultats:

exacts faux exacts

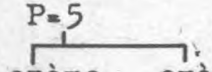

crème crème

fraiche acide

da $=$ dao da ?

$P=5$

$v=f(T)$

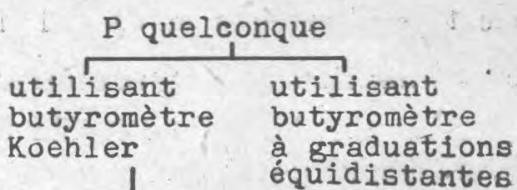

$\mathrm{T}=\mathrm{T}_{\mathrm{I}} \frac{(\mathrm{v}+\mathrm{m}) \mathrm{dax} I 00}{\mathrm{IO0}}$

(formule générale exacte si elle tient compte des variations de da) 
seulement et les calculs étudiés théoriquement ne peuvent être mis en cause ni vérifiés expérimentalement.

L'obtention des résultats par simple lecture sans abaque de correction exige l'emploi de butyromètres gradués spécialement dans lesquels ont introduit soit un volume détermiré (méthode Koehler originale) soit un poids constant de crème (méthode Roeder).

Les résultats ainsi obtenus ne sont exacts que dans la mesure ou le da de l'extraction est identique au da de la graduation.

L'emploi d'abaques de correction tenant compte des variations des différents fácteurs et en particulier de da permettrait d'obtenir des résultats exacts avec n'importe quel type de butyromètre et en analysant un poids quelconque d'échantillon.

L'établissement de telles abaques n'est possible que par la détermination des caractéristiques des facteurs intervenant dans. l'équation générale de la teneur :

$$
\mathrm{T}=\frac{(\mathrm{v}+\mathrm{m}) \mathrm{da} \times 100}{\mathrm{P}}
$$

c'est-à-dire :

10 De da densité apparente de la matière grasse extraite ellemême fonction (équation $\mathrm{n}^{0} 1$ ) :

a) De la densité effective du corps gras extrait;

b) De la quantité de matière grasse pure contenue par gramme de corps gras extrait ;

c) Du taux d'extraction de la matière grasse pure par la. méthode dans le cas considéré ;

$2^{\circ} \mathrm{Du}$ volume de la matière grasse séparée qui se compose :

a) Du volume lu contenu entre les graduations du butyromètre utilisé ;

b) Du volume non compris dans la lecture.

Une deuxième partie consacrée aux recherches de laboratoire proposera des méthodes d'investigation pour l'étude de ces différents facteurs. 